\title{
Precision Measurement of the Monthly Carbon and Oxygen Fluxes in Cosmic Rays with the Alpha Magnetic Spectrometer on the International Space Station
}

\section{Federico Donnini*广}

INFN Sez. Perugia, SSDC-ASI

E-mail: federico.donnini@cern.ch

\begin{abstract}
The precision measurements of the monthly carbon and oxygen cosmic ray fluxes for the period from May 2011 to May 2018 with Alpha Magnetic Spectrometer on the International Space Station are presented. This period covers the ascending phase of solar cycle 24 together with the reversal of the Sun's magnetic field polarity through the maximum. The detailed variations with time of the carbon and oxygen fluxes are shown up to rigidities of $60 \mathrm{GV}$. The time dependence of the carbon to oxygen flux ratio is also presented, together with the flux ratios to the more abundant species proton and helium.
\end{abstract}

36th International Cosmic Ray Conference -ICRC2019-

July 24th - August 1st, 2019

Madison, WI, U.S.A.

* Speaker.

${ }^{\dagger}$ On behalf of the AMS Collaboration. 


\section{Introduction}

Cosmic rays (CR) are charged and neutral particles with galactic or extra-galactic origin reaching the Earth atmosphere from all directions spanning a wide energy range (from few $\mathrm{MeV}$ up to $10^{20} \mathrm{eV}$ ). Cosmic rays entering the heliosphere are subject to diffusion, convection, adiabatic energy losses, and magnetic drift. These processes are strongly correlated with the solar activity. The temporal evolution the solar activity leads to a variation of the cosmic ray intensity measured near Earth, called Solar Modulation (SM) effect. The solar activity has several cycles [?]. The most significant is the 11-year solar cycle during which the number of sunspots changes from minimum to maximum and then back to a minimum. Another is the 22-year cycle of the Sun's magnetic field polarity, which reverses every 11 years during the maxima of the solar cycle. In order to have a correct understanding of CR spectra outside the heliosphere, the SM should be well known and taken into account. Since the time dependent cosmic ray transport in the heliosphere, according to the Parker equation [?], is rigidity dependent and related to changes in solar activity, time correlations at low rigidity among different particle spectra (p, He, C, O) are expected by propagation models. The precise knowledge of the CR fluxes time evolution is needed in order to develop and test different models of the SM effects based on the interaction of cosmic rays with the Heliosphere. AMS-02 already provided important measurement of the time evolution of $\mathrm{p}$ and He spectra, showing discrepancies in their time behavior starting from February 2015. Before AMS data, several effects had been proposed that lead to a time dependence of the $\mathrm{p} / \mathrm{He}$ flux ratio at low rigidities, such as velocity dependence of the diffusion tensor, differences in the interstellar spectra of $p$ and $\mathrm{He}$, and the $3 \mathrm{He}$ and $4 \mathrm{He}$ isotopic composition [?][?][?]. A detailed study of the $\mathrm{C}$ and $\mathrm{O}$ fluxes time evolution, since they are the closest most abundant species, can help to better understand the origin of such dscrepancies observed on $\mathrm{p}$ and He, improving the knoledge of the interactions and the propagation of charged particles inside the Heliosphere. In this letter, the carbon and oxygen fluxes up to $60 \mathrm{GV}$ and their time evolution during the first 7 year of data collacted by AMS-02 will be presented. The carbon and the Oxygen fluxes are measured for the 96 Bartels rotations (27 days) from May 2011 to May 2018.

\section{Detector}

AMS-02 is a large-acceptance high-energy magnetic spectrometer able to perform precision measurements of particles in the GeV-TeV energy range. The AMS-02 apparatus is composed by different sub-detectors for a complete reconstruction of the particle identity. The core of the detector is the silicon Tracker, composed by 9 layers of of double-sided microstrip silicon sensors, 6 of which (L3 to L8) inside the magnetic field generated by a permanent magnet. The tracker accurately measures the curvature of particles in the magnetic field with resolution of $5.1 \mu$ for carbon and $6.3 \mu$ for oxygen [?], and it allows to derive the rigidity $(R=p c / Z e)$ of charged cosmic rays, with a maximum detectable rigidity (MDR) of $3.7 \mathrm{TV}$ for carbon and $3.4 \mathrm{TV}$ for oxygen over the $3 \mathrm{~m}$ lever arm from L1 to L9, and 1.13 TV over the lever arm from L1 to L8. Each layer of the tracker provides also an independent measurement of the charge $Z$ with a resolution of $\Delta Z / Z=5 \%$ for carbon and $4 \%$ for oxygen. Overall, the inner tracker has a resolution of $\Delta Z / Z=$ $2 \%$ for carbon and $1.5 \%$ for oxygen. Two pairs of plastic scintillators, placed at both ends of 
the tracker, measure the Time of Flight (ToF) of the particle and provide the trigger for the rest of the experiment. An anti-coincidence scintillator system (ACC) gives the veto signal in the trigger for particles entering the apparatus out of the Tracker acceptance, i.e. at large angles or from the sides. The AMS- 02 detector is completed by other three sub-detectors which provide the particle identification capabilities: a Ring Image Cherenkov (RICH) below the magnet to measure of the particle velocity; the Transition Radiation Detector (TRD) placed on top of AMS-02 and an Electromagnetic Calorimeter (ECAL) at the bottom for the accurate distinction between leptons and hadrons. More details on the various sub-detectors can be found in [?] and references therein.

\section{Event Selection}

The AMS-02 detector, with its large acceptance and long exposure time, is able to perform precision measurement of the hadronic component in CRs for nuclei up to Fe (Figure 1 left) and the time evolution of their spectra. In the first 7 years (May the 19th, 2011 to May the 28th, 2017) AMS-02 has collected abount 115 Million cosmic ray events. The collection time includes only those seconds during which the detector was in normal operating conditions, AMS-02 was pointing within $40^{\circ}$ of the local zenith, and the ISS was outside of the South Atlantic Anomaly. The measured rigidity is also required to be greater than 1.2 times the maximum geomagnetic cutoff within the AMS field of view. The cutoff was calculated by backtracing [?] particles from the top of AMS out to 50 Earth's radii using the most recent IGRF geomagnetic model [?].

The identification carbon and oxygen is achieved by requiring charge compatible with $Z=6$ and $Z=8$ rispectively on the tracker L1, inner tracker and upper TOF. The L9 charge measurement is not required, since it significantly reduces the statistic. Additional informations on the event selection can be found in ref. [?]. After selection the event sample contains $8.2 \cdot 10^{6}$ carbon events with a purity of $99 \%$ and $7 \cdot 10^{6}$ oxygen events with a purity $>99.8 \%$.
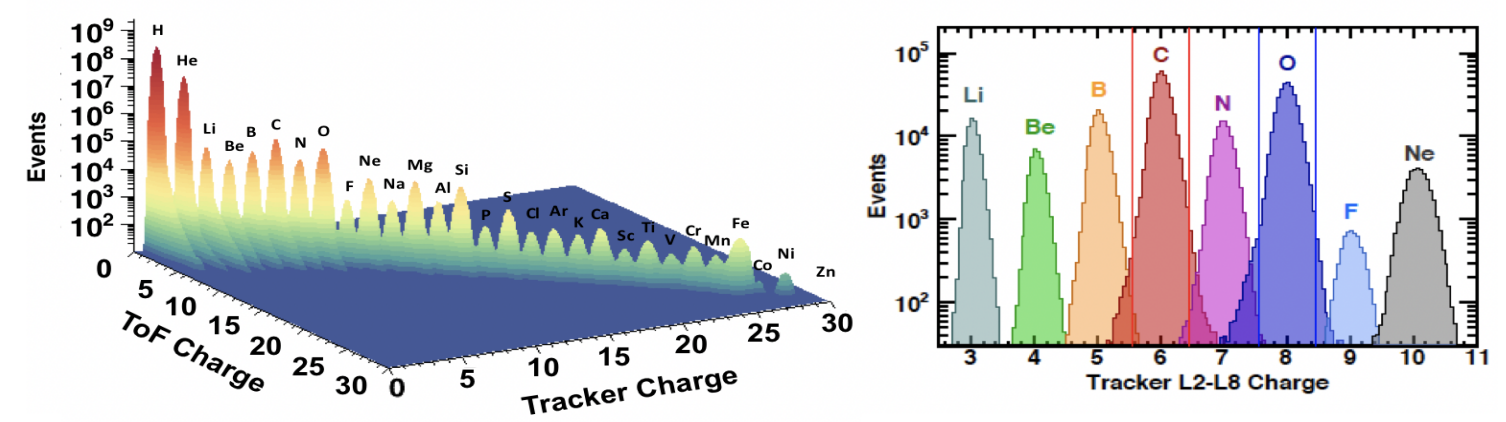

Figure 1: Left: charge measurement provided by Inner Tracker and TOF; AMS-02 is able to perform precise measurement of the hadronic component in CRs for nuclei up to Fe. Right: Distribution of the charge measured with the inner tracker ( $L 2-L 8)$ for samples from $Z=3$ to $Z=10$ selected by the combined charge measured with L1, the upper TOF, and the lower TOF over the rigidities above 4 GV. The colored vertical lines correspond to the charge selection in the inner tracker for carbon (red) and oxygen (blue).

The residual background for carbon and oxygen event samples have two sources. The first one come from the interaction of heavier nuclei in the material between L1 and L2 (TRD and upper TOF). This residual background is evaluated by fitting the charge distribution of tracker L1 
with charge distribution templates of $\mathrm{Li}, \mathrm{Be}, \mathrm{B}, \mathrm{C}, \mathrm{N}$ and $\mathrm{O}$ as shown in Fig 2. The templates are obtained from samples selected to be non-interacting samples at L2 by the use of the charge measurement with L1 and L3-L8. This background is found to be $<0.5 \%$ for carbon and negligible for oxygen over the entire rigidity range. The second source of background come from $\mathrm{N}, \mathrm{O}, \mathrm{F}$, and $\mathrm{Ne}$ interacting in materials above L1 (thin support structures made of carbon fiber and aluminum honeycomb). It has been estimated from simulation using MC samples generated according to AMS flux measurements. This background is $<0.5 \%$ for carbon and negligible for oxygen over the entire rigidity range. The overall uncertainty due to background subtraction is $<0.5 \%$ for carbon and negligible for oxygen over the entire rigidity range.

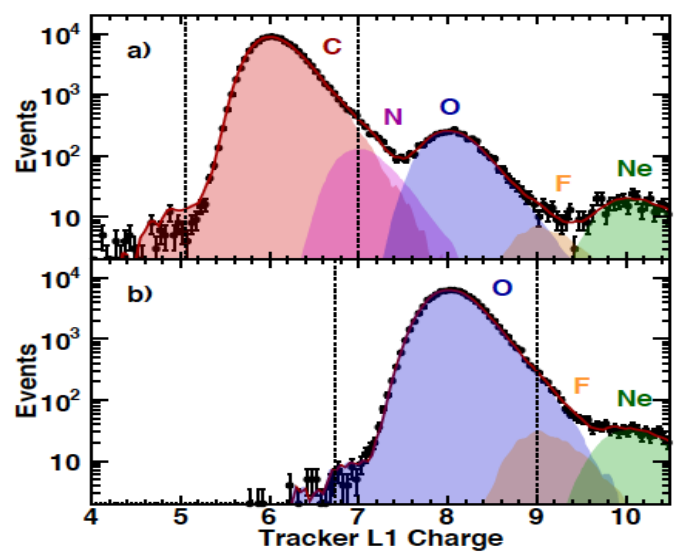

Figure 2: Charge distributions measured by tracker L1 for (a) carbon and (b) oxygen events selected by the inner tracker in the rigidity range between 9 and 11 GV (black dots). The solid red curves show the $t$ to the data of the $C, N, O, F$, and Ne charge distribution templates. The templates are obtained from samples selected to be non-interacting samples at L2 by the use of the charge measurement with L1 and L3-L8. The charge selections applied on tracker L1 are shown as vertical dashed lines. The residual backgrounds to the carbon and oxygen samples are calculated by integrating the charge template distributions over the selection range.

\section{Data Analysis}

The isotropic flux $\Phi_{i}$ during a Bartels rotation in the $i$-th rigidity bin $\left(R_{i}, R_{i}+\Delta R_{i}\right)$ is given by

$$
\Phi_{i}=\frac{N_{i}}{A_{i} \varepsilon_{i} T_{i} \Delta R_{i}}
$$

where, for that Bartels rotation, $N_{i}$ is the number of events rispectively carbon and oxygen corrected for bin-to-bin migrations, $A_{i}$ is the effective acceptance, $\varepsilon_{i}$ is the trigger efficiency, and $T_{i}$ is the collection time. In this contribution, the carbon flux and the oxygen flux are measured in 40 bins from 1.9 to $60 \mathrm{GV}$.

The bin-to-bin migration of events was corrected using the unfolding procedure described in Ref. [?] independently for each Bartels rotation for the carbon and oxygen samples. The trigger efficiencies have been measured to be $>99 \%$ for carbon and oxygen over the entire rigidity range 
in each Bartels rotation. A detailed description of the trigger efficiency evaluation can be found in ref. [?].

The detector acceptance directly relates the number of selected particles to the flux and depends on the detector geometry as well as on the detection and selection efficiencies. The acceptance of AMS-02 has been evaluated using a Monte Carlo simulation (MC) of the entire detector, since an analytical calculation is impractical even for simple detector geometries and neglecting efficiency effects.

The AMS-02 Monte Carlo simulation, based on the GEANT-4.10.1 package [?], contains a detailed description of the detector: its geometry and its composition, with the best possible estimates of the matter density distribution of both active and passive areas in the instrument. The detector description is combined with a simulation of the physical processes that take place inside the detector when a charged particle passes through. Once the various energy depositions and interactions of particles inside the detector are described, the hit simulation produces the experimental signals which set the basis for an event reconstruction, performed in the same way as for the real data. The end result is a data structure similar to the one obtained in the flight data. The simulation is validated comparing the reconstruction and selection efficiencies estimated from flight data with those obtained from the simulation itself: any difference between the two evaluations of each efficiency is used to correct the estimated acceptance and to assess the systematic uncertainty on the measurement.

Since an accurate description of nuclear fragmentation into the detector materials is needed both in the acceptance estimation and in the evaluation of irreducible backgrounds, a detailed study has been performed to verify and tune the description of inelastic nuclear interactions in the MC simulation. Nuclear inelastic cross-sections are simulated in the MC by using the Glauber-Gribov model to define the overall interaction rate, then the resulting secondary products are generated according to the DPMJET 2.5 model [?]. The nuclear interactions description tuning in the MC simulation is described in details in [?] .

The contributions to the systematic errors for each Bartels rotation come from the uncertainties in the two background estimations, the geomagnetic cutoff factor, the acceptance calculation, the rigidity resolution function and the absolute rigidity scale. These systematic errors are time independent.

The systematic errors coming from the geomagnetic cutoff is evaluated by varing the cutoff factor from 1.0 to 1.4 , and it is found to be $<0.1 \%$ in the rigidity range below $30 \mathrm{GV}$.

The systematic error due to the rigidity resolution functions was obtained by repeating the unfolding procedure while varying the widths of the Gaussian cores of the resolution functions by $5 \%$ and by independently varying the amplitudes of the non-Gaussian tails by $10 \%$. The resulting systematic error on the fluxes is less than $1 \%$ for both $\mathrm{C}$ and $\mathrm{O}$.

The systematic uncertainty on the rigidity scale arises from the residual tracker misalignment and from the magnetic field map measurement [?]. Another energy measurement is needed to assess this uncertainty, for this reason a sample of electrons and positrons has been used, since the lepton energy estimated with the ECAL is precise enough to be compared with the tracker rigidity measurement. The error on carbon and oxygen fluxes due to uncertainty on the rigidity scale is $<1 \%$. 
Finally, the time dependent systematic errors due to the variations of trigger and reconstruction efficiency for different Bartels rotations are still under study.

\section{Results}

Figure 3(left) shows the detailed behavior of the $\mathrm{C}$ and $\mathrm{O}$ fluxes for 5 characteristic rigidity bins. Both the $\mathrm{C}$ and $\mathrm{O}$ fluxes, as seen for $\mathrm{p}$ and $\mathrm{He}$ [?], exhibit large variations with time at low rigidities which decrease with increasing rigidity and vanish above $30 \mathrm{GV}$. The time behavior of $\mathrm{C}$ and $\mathrm{O}$ fluxes are similar; this is confirmed on Figure 3(right), which shows the costant time behavior of the $\mathrm{C} / \mathrm{O}$ ratio for the 5 characteristic rigidity bins.
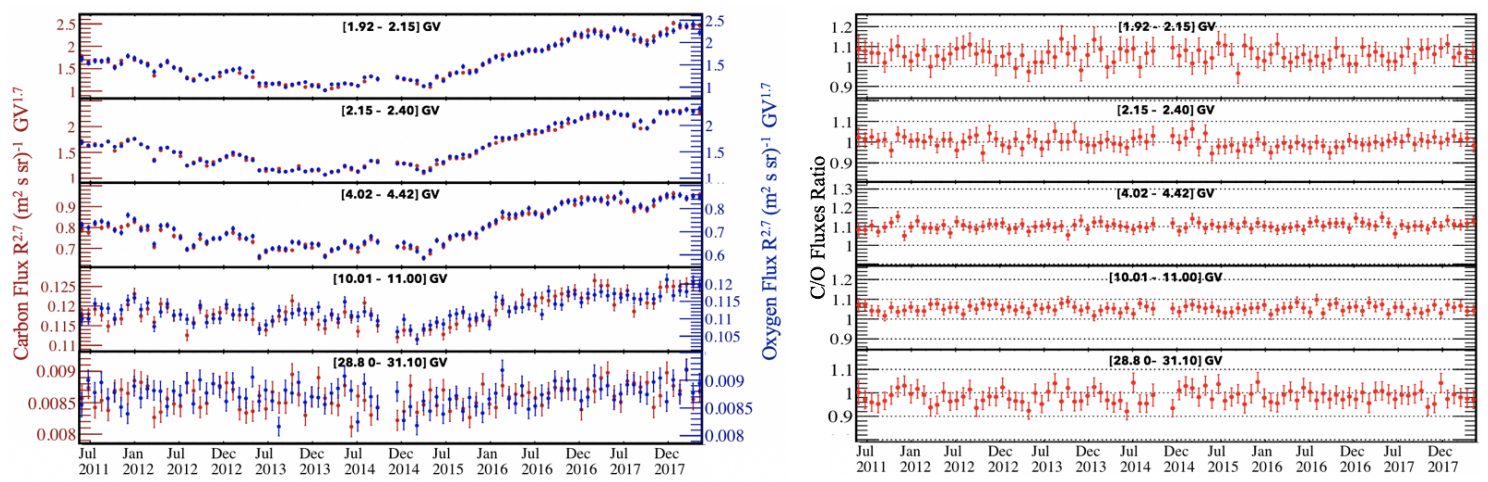

Figure 3: Left: the AMS carbon (red, left axis) and oxygen (blue, right axis) fluxes multiplied by $R^{2.7}$ with the statistical uncertainties, as a function of time from May 2011 up to May 2018, for 5 rigidity bins. Right: the AMS C/O ratio with the statistical uncertainties, as a function of time from May 2011 up to May 2018.

In order to compare the time behavior of the carbon and oxygen fluxes, the ratio with respect p and He from May 2011 up to May 2017 has been performed. Figure 4(left) shows rispectively the $\mathrm{C} / \mathrm{p}$ ratio (on top) and the $\mathrm{O} / \mathrm{p}$ ratio (on bottom), for the first rigidity interval (which is the most influenced by the SM), with the statistical uncertainties, as a function of time. Even if the fluxes are strongly influeced by the statistical fluctuations, the time behavior of proton, carbon and oxygen seems to be similar, and their ratio is time independent for all the rigidity bins. From the other side, instead, as shown in Figure 4(right) the $\mathrm{C} / \mathrm{He}$ ratio (on top) and the $\mathrm{O} / \mathrm{He}$ ratio (on bottom) present a small decreasing behavior coinciding with the period during which the fluxes start to rise. In order to better understand these behavior, several efforts to reduce the statistical fluctuation on the $\mathrm{C}$ and $\mathrm{O}$ fluxes in still ongoing.

\section{Conclusions}

A detailed study of $\mathrm{C}$ and $\mathrm{O}$ fluxes and their time evolution can help to better understand the discrepancies observed between $\mathrm{p}$ and $\mathrm{He}$, in order to test and improve the knowledge of the propagation and the interactions of charged particles inside the Heliosphere. AMS-02 measured the time evolution of $\mathrm{C}$ and $\mathrm{O}$ fluxes during the ascending phase of solar cycle 24 through its maximum and toward its minimum, using the data collected in 7 year of operation. The precision measurement of $\mathrm{C}$ and $\mathrm{O}$ fluxes show large variations with time at low rigidities which decrease 

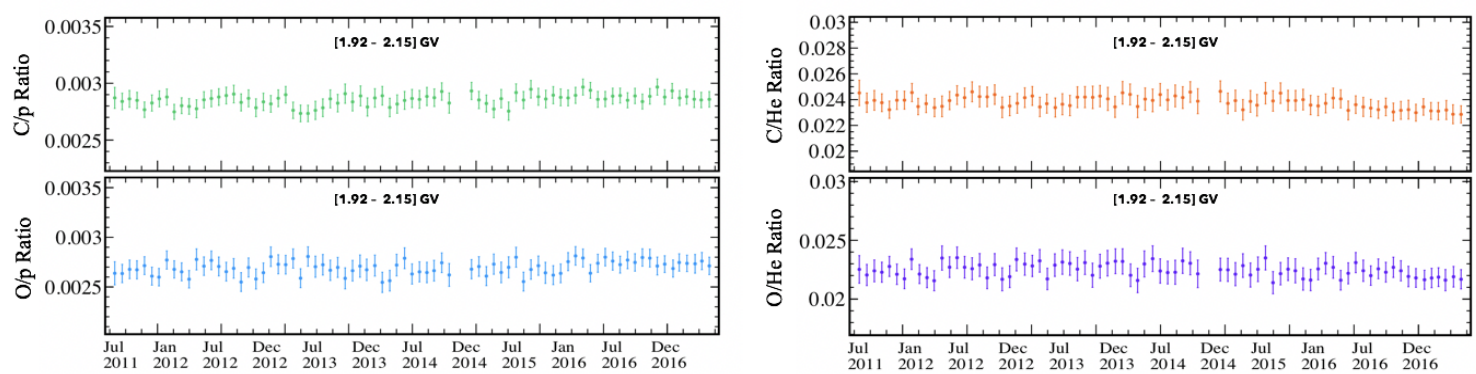

Figure 4: Left: the AMS C/p (on top) and O/p (on bottom) fluxes ratios with the statistical uncertainties, as a function of time from May 2011 up to May 2017, for the first rigidity interval. The ratios are costant in time. Right: the AMS C/He (on top) and O/He (on bottom) fluxes ratios with the statistical uncertainties, as a function of time from May 2011 up to May 2017, for the first rigidity interval. The ratios, show a decreasing behavior starting from Feb 2015.

with increasing rigidity and vanish above $30 \mathrm{GV}$. These results show also a similar time behavior observed on proton, with discrepancies with respect the He behavior coinciding with the period during which the fluxes start to rise. In order to better investigate and improve these results, several efforts are still ongoing.

\section{References}

\section{References}

[1] M. S. Potgieter, Living Rev. Solar Phys., 10, 3 (2013)

[2] E. N. Parker, Planet. Space Sci., 13, 9 (1965)

[3] I. Cholis, D. Hooper, and T. Linden, Phys. Rev, D 93, 043016 (2016)

[4] G. Gloeckler, J.R. Jokipii, Phys. Rev. Lett., 17, 203,(1966)

[5] N. Tomassetti, M. Orcinha, F. Barão, and B. Bertucci, Astrophys. J. Lett., 849, L32 (2017)

[6] G. Ambrosi, V. Choutko, C. Delgado, A. Oliva, Q. Yan, and Y. Li, Nucl. Instrum. Methods Phys. Res., Sect. A 869, 29 (2107)

[7] M. Aguilar et al., Phys. Rev. Lett., 110, 141102 (2013)

[8] J. Alcaraz et al., Phys. Lett., B 183, 1216 (2016)

[9] C. Finlay et al., Geophys. J. Int., 484, 10 (2000)

[10] M. Aguilar et al., Phys. Rev. Lett., 119, 251101 (2017)

[11] M. Aguilar et al., Phys. Rev. Lett. 114, 171103 (2015)

[12] M. Aguilar et al., Phys. Rev. Lett. 115, 211101 (2015)

[13] J. Allison et al., Nucl. Instrum. Methods Phys. Res., Sect. A 835, 186 (2016); S. Agostinelli et al., Nucl. Instrum. Methods Phys. Res., Sect. A 506, 250 (2003)

[14] M. Aguilar et al., Phys. Rev. Lett., 121, 051101 (2018) 